\title{
Endotrivial modules over groups with quaternion or semi-dihedral Sylow 2-subgroup
}

Received April 22, 2010

\begin{abstract}
Let $G$ be a finite group with a Sylow 2-subgroup $P$ which is either quaternion or semidihedral. Let $k$ be an algebraically closed field of characteristic 2 . We prove the existence of exotic endotrivial $k G$-modules, whose restrictions to $P$ are isomorphic to the direct sum of the known exotic endotrivial $k P$-modules and some projective modules. This provides a description of the group $T(G)$ of endotrivial $k G$-modules.
\end{abstract}

\section{Introduction}

Suppose that $G$ is a finite group and that $k$ is a field of characteristic $p$. Endotrivial $k G$ modules appear in a natural way in many areas surrounding local analysis of finite groups. They were introduced by Dade [15] who classified them in the case where $G$ is an abelian $p$-group. A complete classification of endotrivial modules over the modular group rings of $p$-groups was completed just a few years ago $[6,11,12,13]$. The class of all endotrivial modules for a given group $G$ gives rise to an abelian group $T(G)$ (with respect to the tensor product). This group is finitely generated and carries with it all of the information of the classification. The group $T(G)$ is of interest because it is an important part of the Picard group of self-equivalences of the stable category of finitely generated $k G$-modules. The so-called self-equivalences of Morita type are induced by tensoring with endotrivial modules. For this reason, it is of interest to extend the classification beyond $p$-groups to general finite groups. Some progress has been made in that direction [7, 8, 9, 10, 22].

In this paper we consider two out-lying situations where the answer to a different sort of problem is sought. In the classification of endotrivial modules over $p$-groups, there are exactly two cases in which the group $T(P)$ of endotrivial modules for a noncyclic $p$ group $P$ has torsion elements. The two cases occur when $p=2$ and $P$ is either quaternion (meaning ordinary or generalized quaternion) or semi-dihedral. For a group $G$ having

J. F. Carlson: Department of Mathematics, University of Georgia, Athens, GA 30602, USA; e-mail: jfc@math.uga.edu

N. Mazza: Department of Mathematics and Statistics, Lancaster University, Lancaster LA1 4YF, England; e-mail: n.mazza@lancaster.ac.uk

J. Thévenaz: Section de mathématiques, EPFL, Station 8, CH-1015 Lausanne, Switzerland; e-mail: Jacques.Thevenaz@epfl.ch

Mathematics Subject Classification (2010): Primary 20C20. 
such a $P$ as its Sylow 2-subgroup, the question is whether the restriction map $T(G) \rightarrow$ $T(P)$ is surjective. Specifically, we need to know if the torsion elements in $T(P)$ are in the image of the restriction. Do these modules lift or extend in some way from $P$ to $G$ ? In this paper we show that the answer is yes, the restriction map is surjective. In the course of the investigation we are able to find much more information about the structure of $T(G)$ and about the modules themselves. The only other case in which $T(P)$ has torsion elements occurs when $P$ is cyclic, and this case was treated in [22].

It is somewhat surprising that the two cases require very different methods. In the situation where the Sylow 2-subgroup $P$ of $G$ is quaternion and the unique involution in $P$ is central in $G$, we use a general method for finding exotic endotrivial modules as subquotients of $\Omega^{2}(k)$, the second syzygy of the the trivial module $k$. This method has been used in earlier papers $[6,8]$. There are two means for extending this result to general groups with quaternion Sylow 2-subgroups. One involves invoking the Brauer-Suzuki Theorem [5] on the structure of such groups. The more elementary method is to note that the centralizer of the involution of $P$ is a strongly 2-embedded subgroup of $G$ and we can apply a theorem of [22]. These results appear in Sections 3 and 4, after a general introduction to endotrivial modules in Section 2. Moreover, we prove in Section 5 that there are always torsion endotrivial modules which are uniserial.

By contrast, the key to the semi-dihedral case is the theory of Auslander-Reiten sequences or almost split sequences. In Section 6, we construct exotic endotrivial modules over finite groups having a semi-dihedral Sylow 2-subgroup, by using a certain Auslander-Reiten sequence which has as middle term the heart $\operatorname{Rad}\left(R_{k}\right) / \operatorname{Soc}\left(R_{k}\right)$ of the projective cover $R_{k}$ of the trivial module. The existence of this sequence is related to the fact that the component of the stable Auslander-Reiten quiver containing $\Omega(k)$ has tree class $D_{\infty}$. This is an important result due to K. Erdmann [20, 18, 19]. The fact that the end terms of this Auslander-Reiten sequence are endotrivial is due to C. Bessenrodt [3].

\section{Preliminaries}

Throughout this paper, we let $k$ denote an algebraically closed field of prime characteristic $p$. From Section 3 onwards, we will assume that $p=2$. In addition, we assume that all modules are finitely generated. In this section, we briefly recap some needed basics.

Given a finite group $H$, we write $k$ for the trivial $k H$-module, or, whenever $H$ needs to be clarified, we write $k_{H}$ instead. Unless otherwise specified, the symbol $\otimes$ is the tensor product $\otimes_{k}$ of the underlying vector spaces, and in the case of $k H$-modules, $H$ acts diagonally on the factors. If $M$ is a $k H$-module, and $\varphi: Q \rightarrow M$ its projective cover, then we let $\Omega(M)$ denote the kernel of $\varphi$. Likewise, if $\vartheta: M \rightarrow Q$ is the injective hull of $M$ (recall that $k H$ is a self-injective ring so $Q$ is also projective), then $\Omega^{-1}(M)$ denotes the cokernel of $\vartheta$. Inductively, with $\Omega^{1}(M)=\Omega(M)$, we set $\Omega^{n}(M)=\Omega\left(\Omega^{n-1}(M)\right)$ and $\Omega^{-n}(M)=\Omega^{-1}\left(\Omega^{-n+1}(M)\right)$ for all integers $n>1$.

If $G$ is a finite group of order divisible by $p$, then a $k G$-module $M$ is endotrivial if its endomorphism algebra $\operatorname{End}_{k}(M)$ is isomorphic (as a $k G$-module) to the direct sum of the trivial module $k_{G}$ and a projective $k G$-module. In other words, a $k G$-module $M$ is 
endotrivial if and only if $M^{*} \otimes M \cong k \oplus(\operatorname{proj})$, where $M^{*}$ denotes the $k$-dual $\operatorname{Hom}_{k}(M, k)$ of $M$, and (proj) some projective module.

Lemma 2.1. Let $G$ be a finite group of order divisible by $p$.

(1) Let $M$ be a kG-module. If $M$ is endotrivial, then $M$ splits as the direct sum $M_{\diamond} \oplus$ (proj) for an indecomposable endotrivial $k G$-module $M_{\diamond}$, which is unique up to isomorphism.

(2) The relation

$$
M \sim N \Leftrightarrow M_{\diamond} \cong N_{\diamond}
$$

on the class of endotrivial $k G$-modules is an equivalence relation. We let $T(G)$ be the set of equivalence classes. Every equivalence class contains a unique indecomposable module up to isomorphism.

(3) The tensor product induces an abelian group structure on the set $T(G)$ by

$$
[M]+[N]=[M \otimes N] .
$$

The zero element of $T(G)$ is the class $[k]$ of the trivial module, consisting of all modules of the form $k \oplus$ (proj). The inverse of the class of a module $M$ is the class of the dual module $M^{*}$.

The group $T(G)$ is called the group of endotrivial $k G$-modules. It is known to be a finitely generated abelian group. In particular, the torsion subgroup $T T(G)$ of $T(G)$ is finite. The torsion-free rank of $T(G)$ can be described explicitly (see [8]).

We often use the following fact (see [11, Lemma 2.9]).

Lemma 2.2. For a $k G$-module $M$, if the restriction of $M$ to every elementary abelian p-subgroup of $G$ is an endotrivial module, then $M$ is an endotrivial module.

We use the following easy result.

Lemma 2.3. Let $P$ be a Sylow $p$-subgroup of $G$ and let $M$ be an endotrivial $k G$-module.

(1) If $p$ is odd, then $\operatorname{Dim}(M) \equiv \pm 1(\bmod |P|)$.

(2) If $p=2$, then $\operatorname{Dim}(M) \equiv \pm 1(\bmod |P| / 2)$.

Proof. By the very definition, we have $\operatorname{Dim}(M)^{2}=\operatorname{Dim}\left(\operatorname{End}_{k}(M)\right)=1+n$, where $n$ is the dimension of a projective module. Since a projective module is free on restriction to $P$, its dimension must be a multiple of $|P|$. Hence

$$
\operatorname{Dim}(M)^{2} \equiv 1(\bmod |P|) .
$$

Thus $\operatorname{Dim}(M)$ is a square root of 1 modulo $|P|$ and the result follows.

When $p=2$, the congruence $\operatorname{Dim}(M) \equiv|P| / 2 \pm 1(\bmod |P|)$ does not happen very often, but it does occur when $P$ is either quaternion or semi-dihedral. We shall say that an endotrivial $k G$-module $M$ is exotic if $M$ is indecomposable and if $\operatorname{Dim}(M) \equiv|P| / 2+1$ $(\bmod |P|)$, where $P$ is a Sylow 2-subgroup of $G$ (and $p=2$ of course).

The motivation for the present research stems from the classification of endotrivial modules over finite $p$-groups. The results we need are summarized as follows. 
Theorem 2.4. Let $P$ be a nontrivial finite p-group.

(1) If $P$ is cyclic of order $\geq 3$, then $T(P) \cong \mathbb{Z} / 2 \mathbb{Z}$. If $P$ is cyclic of order 2 , then $T(P)=\{0\}$.

(2) If $P$ is generalized quaternion, then $T(P) \cong \mathbb{Z} / 2 \mathbb{Z} \oplus \mathbb{Z} / 4 \mathbb{Z}$. The summand $\mathbb{Z} / 2 \mathbb{Z}$ is generated by the class of an indecomposable endotrivial module $U$ which is exotic and self-dual. The second summand is generated by the class of the syzygy $\Omega(k)$ of the trivial module, which has order 4 .

(3) If $P$ is semi-dihedral, then $T(P) \cong \mathbb{Z} / 2 \mathbb{Z} \oplus \mathbb{Z}$. The summand $\mathbb{Z} / 2 \mathbb{Z}$ is generated by the class of an indecomposable endotrivial module $U$ which is exotic and selfdual. The second summand is generated by the class of the syzygy $\Omega(k)$ of the trivial module, which has infinite order.

(4) If $P$ is not cyclic, generalized quaternion, or semi-dihedral, then $T(P)$ is torsion-free.

Statement (1) is easy (see [15]). Statement (2) is proved in [11], and also implicitly in [16]. Statement (3) is proved in [11], while (4) is one of the main results in [13].

Remark 2.5. If $P$ is a semi-dihedral group, then the module $U$ in statement (3) is unique up to isomorphism, because $T T(P) \cong \mathbb{Z} / 2 \mathbb{Z}$, so $U$ is the only nontrivial indecomposable endotrivial module such that $U \cong U^{*}$. Moreover, $\Omega^{2 n}(U)$ is again exotic for every $n \in \mathbb{Z}$, but not self-dual unless $n=0$.

Remark 2.6. If $P$ is a quaternion group, then there are two possible exotic generators for the summand of $T(P)$ isomorphic to $\mathbb{Z} / 2 \mathbb{Z}$ in statement (2), namely, $U$ and $\Omega^{2}(U)$. The subgroup of elements of order 2 in $T(P)$ is a Klein four group $\mathbb{Z} / 2 \mathbb{Z} \oplus \mathbb{Z} / 2 \mathbb{Z}$, generated by $[U]$ and $\left[\Omega^{2}(k)\right]$. There are three elements of order 2 , one of them being $\left[\Omega^{2}(k)\right]$. The other two are the classes of the two exotic modules $U$ and $\Omega^{2}(U)$. Note that we have $\left[\Omega^{2}(U)\right]=[U]+\left[\Omega^{2}(k)\right]$ in $T(P)$. The modules $\Omega^{i}(k)($ for $1 \leq i \leq 3)$ have dimension $|P| \pm 1$, the two exotic $k P$-modules $U$ and $\Omega^{2}(U)$ have dimension $|P| / 2+1$, and the two remaining indecomposable endotrivial $k P$-modules (which are actually $\Omega(U)$ and $\left.\Omega^{3}(U)\right)$ have dimension $|P| / 2-1$.

We end this section with a review of a few facts about support varieties that will be needed, particularly in the next section.

The cohomology ring $\mathrm{H}^{*}(G, k)$ is a finitely generated, graded commutative $k$-algebra and has a maximal ideal spectrum $V_{G}(k)$ which is a homogeneous affine variety. If $M$ is a finitely generated $k G$-module, then its cohomology $\operatorname{ring} \operatorname{Ext}_{k G}^{*}(M, M)$ is a finitely generated module over $\mathrm{H}^{*}(G, k)$, and we let $J(M)$ denote its annihilator in $\mathrm{H}^{*}(G, k)$. The support variety of $M$ is the set $V_{G}(M)=V_{G}(J(M)) \subseteq V_{G}(k)$ of all maximal ideals that contain $J(M)$. Hence, $V_{G}(M)$ is a closed homogeneous subvariety of $V_{G}(k)$. The support varieties have some important properties. The properties were developed by many people. Proofs and history can be found in the standard references [2, 14].

One of the most valuable tools in the theory of module varieties is the theorem of Quillen which says that

$$
V_{G}(k)=\bigcup_{E \in \mathcal{E} \mathcal{A}} \operatorname{res}_{G, E}^{*}\left(V_{E}(k)\right)
$$


where $\mathcal{E} \mathcal{A}$ is the collection of all elementary abelian $p$-subgroups of $G$. A consequence of this theorem is the theorem of Chouinard which states that a finitely generated $k G$ module $M$ is projective if and only if it is projective on restriction to every elementary abelian $p$-subgroup of $G$. This result is included in the theorem that follows. This theorem presents most of the properties of support varieties that will be needed in the paper.

Theorem 2.7. Suppose that $L, M$ and $N$ are $k G$-modules.

(1) The module $M$ is projective if and only if $V_{G}(M)=\{0\}$.

(2) A $k G$-module $M$ is projective if and only if its restriction to every elementary abelian p-subgroup of $G$ is projective.

(3) $V_{G}\left(M^{*}\right)=V_{G}\left(\Omega^{n}(M)\right)=V_{G}(M)$ for any integer $n$.

(4) $V_{G}(M \oplus N)=V_{G}(M) \cup V_{G}(N)$.

(5) $V_{G}(M \otimes N)=V_{G}(M) \cap V_{G}(N)$.

(6) If the sequence $0 \rightarrow L \rightarrow M \rightarrow N \rightarrow 0$ is exact, then $V_{G}(M) \subseteq V_{G}(L) \cup V_{G}(N)$.

(7) $V_{G}(M)=\bigcup_{E \in \mathcal{E} \mathcal{A}} \operatorname{res}_{G, E}^{*}\left(V_{E}(M)\right)$.

(8) Suppose that $V_{G}(M)=V_{1} \cup V_{2}$ where $V_{1}$ and $V_{2}$ are closed sets such that $V_{1} \cap V_{2}$ $=\{0\}$. Then $M \cong M_{1} \oplus M_{2}$ where $V_{G}\left(M_{1}\right)=V_{1}$ and $V_{G}\left(M_{2}\right)=V_{2}$.

(9) Suppose that $\zeta \in \mathrm{H}^{n}(G, k)$, and let $\hat{\zeta}: \Omega^{n}(k) \rightarrow k$ be a cocycle representing $\zeta$. Let $L_{\zeta}$ denote the kernel of $\hat{\zeta}$. Then $V_{G}\left(L_{\zeta}\right)=V_{G}(\zeta)$.

\section{The second syzygy of the trivial module}

In this section we analyze the structure of the second syzygy $\Omega^{2}(k)$ of the trivial module for a finite group $G$ with a quaternion Sylow 2-subgroup $P$. We assume that the unique involution $z$ of $P$ is central in $G$. This assumption is required by the methods that we use. However, statements made about $\Omega^{2}(k)$ or about any module in the principal block of $k G$ hold without the assumption on the centrality of $z$, because we know from the BrauerSuzuki Theorem [5] that the image of $z$ is central in $G / O_{2^{\prime}}(G)$ and $O_{2^{\prime}}(G)$ is the kernel of the principal block of $k G$. In addition, from now on, we assume that the characteristic of $k$ is 2 .

We set $\bar{G}=G /\langle z\rangle$ and $\bar{H}=H /\langle z\rangle$ for any subgroup $H$ of $G$ containing $z$. We also write $\bar{x}$ for the image of $x \in G$ in $\bar{G}$. For a $k G$-module $V$, let

$$
V_{0}=\{v \in V \mid(z-1) v=0\} .
$$

Note that $V_{0}$ is a $k \bar{G}$-module. Moreover, $V_{0}$ contains the submodule $(z-1) V$ and multiplication by $z-1$ induces an isomorphism $V / V_{0} \cong(z-1) V$.

Applying this to the module $M=\Omega^{2}(k)$, we notice that

$$
M \downarrow_{\langle z\rangle}^{G} \cong \Omega^{2}\left(k_{\langle z\rangle}\right) \oplus R=k_{\langle z\rangle} \oplus R
$$

where $R$ is a projective $k\langle z\rangle$-module. Since $(z-1) R=R_{0}$, we deduce that $M_{0} /(z-1) M$ is one-dimensional. Thus $M=\Omega^{2}(k)$ has a filtration

$$
\{0\} \subset(z-1) M \subset M_{0} \subset M
$$


with the top $M / M_{0}$ isomorphic to the bottom $(z-1) M$ and with a one-dimensional middle module $M_{0} /(z-1) M$ (which is actually the trivial module, see Proposition 3.5).

Proposition 3.1. Suppose that $P$ is a quaternion 2-group. Let $M=\Omega^{2}(k)$. Then $M_{0} \cong$ $\Omega^{2}(k \bar{P})$ as $k \bar{P}$-modules.

Proof. This result could be proved by exhibiting a presentation for the module $\Omega^{2}(k)$ and meticulously constructing an isomorphism. However, we prove the result using more theoretical methods which illuminate some of the ideas in this paper.

First suppose that $|P|=8$, so that $\bar{P}$ is a Klein four group. Then $\operatorname{Dim}(M)=9$ and because the restriction of $M$ to $\langle z\rangle$ is the direct sum of a trivial module and four copies of $k\langle z\rangle$, we see that $\operatorname{Dim}\left(M_{0}\right)=5$. Also $\operatorname{Dim}\left(\operatorname{Soc}\left(M_{0}\right)\right)=\operatorname{Dim}(\operatorname{Soc}(M))=2$, and $M_{0}$ has no nonzero free $k \bar{P}$-direct summand, because such a summand would lift to a free direct summand of $M$ as a $k P$-module (by Proposition 4.2 below), but $M$ is indecomposable. By the classification of the indecomposable modules over a Klein four group (see [2, Theorem 4.3.3]), we know that $\Omega^{2}\left(k_{\bar{P}}\right)$ is, up to isomorphism, the only indecomposable $k \bar{P}$-module whose dimension is 5 and whose socle has dimension 2 . So we need only show that $M_{0}$ is indecomposable.

So assume that $M_{0}$ decomposes. If $M_{0}$ had a 4-dimensional direct summand, it could not be free, hence the dimension of its socle would be at least 2 and so $\operatorname{Dim}(\operatorname{Soc}(M)) \geq 3$, which is a contradiction. Thus $M_{0}$ would be a direct sum of a module of dimension 3 (which is isomorphic to $\Omega\left(k_{\bar{P}}\right)$ ) and a module of dimension 2. But $M_{0}$ is defined over $\mathbb{F}_{2}$ and the decomposition must also exist over $\mathbb{F}_{2}$. However, every indecomposable 2-dimensional $\mathbb{F}_{2} \bar{P}$-module has the form $\mathbb{F}_{2} \uparrow \frac{\bar{P}}{H}$ for $\bar{H}$ a subgroup of index 2 in $\bar{P}$. In particular, on restriction to $\bar{H}$, this 2-dimensional module is the direct sum of two trivial modules.

Now $\bar{H}=\langle\bar{x}\rangle$ for some element $x$ of order 4 in $P$. Since $H=\langle x\rangle$ is cyclic, $\Omega^{2}\left(k_{H}\right) \cong k_{H}$, and therefore $M \downarrow_{H}^{P}$ is the direct sum of a trivial module and two copies of $k H$. It follows that $M_{0} \downarrow \frac{\bar{P}}{H}$ is the direct sum of a trivial module and two copies of $k \bar{H}$. Therefore, we cannot have two trivial modules as direct summands and the proposed decomposition of $M_{0}$ is not possible. This completes the proof in the case where $|P|=8$.

Assume now that $|P|>8$. In this situation, $\bar{P}$ is a dihedral group and $P$ has two quaternion subgroups $E_{1}$ and $E_{2}$ of order 8 such that $\bar{E}_{1}$ and $\bar{E}_{2}$ are representatives of the two conjugacy classes of maximal elementary abelian 2-subgroups of $\bar{P}$. Let $E$ be either $E_{1}$ or $E_{2}$. The restriction of $M$ to a $k E$-module has the form

$$
M \downarrow_{E}^{P} \cong \Omega^{2}\left(k_{E}\right) \oplus F
$$

where $F$ is a free $k E$-module. Since $E$ is quaternion of order 8 , it follows from the first part of the proof that the restriction of $M_{0}$ to $\bar{E}$ has the form

$$
M_{0} \downarrow \frac{\bar{P}}{E} \cong \Omega^{2}\left(k_{\bar{E}}\right) \oplus(z-1) F .
$$

Here, $(z-1) F$ is a free $k \bar{E}$-module. Consequently, $M_{0}$ is an endotrivial $k \bar{P}$-module since its restriction to every elementary abelian 2-subgroup is an endotrivial module (see Lemma 2.2). By the classification of endotrivial modules over dihedral 2-groups [11], 
$M_{0} \cong \Omega^{2}\left(k_{\bar{P}}\right) \oplus Q$ for some projective module $Q$. However, the dimension of $M_{0}$ is $|P| / 2+1$, which is also the dimension of $\Omega^{2}\left(k_{\bar{P}}\right)$. Hence $Q=0$ and $M_{0} \cong \Omega^{2}\left(k_{\bar{P}}\right)$.

Continuing with the module $M=\Omega^{2}(k)$ as in Proposition 3.1, we require also some further information on the decomposition of the $k \bar{P}$-module $(z-1) M$, which is a maximal submodule of $M_{0}$.

Proposition 3.2. Suppose that $P$ is a quaternion 2-group. Let $M=\Omega^{2}(k)$.

(1) The module $(z-1) M$ decomposes as $(z-1) M \cong N_{1} \oplus N_{2}$ with $\operatorname{Dim}\left(N_{1}\right)=$ $\operatorname{Dim}\left(N_{2}\right)=|P| / 4$.

(2) $N_{1}$ and $N_{2}$ are indecomposable.

(3) If $|P|=8$, then the support varieties $V_{\bar{P}}\left(N_{1}\right)$ and $V_{\bar{P}}\left(N_{2}\right)$ are distinct lines in $V_{\bar{P}}(k) \cong k^{2}$.

(4) If $|P|>8$, then $V_{\bar{P}}\left(N_{1}\right)$ and $V_{\bar{P}}\left(N_{2}\right)$ are lines in the two different components of the variety $V_{\bar{P}}(k)$. In particular, $N_{1}$ is free on restriction to any element of one of the conjugacy classes of maximal elementary abelian subgroups, and $N_{2}$ is free on restriction to any elementary abelian subgroup in the other conjugacy class.

Proof. Suppose first that $|P|=8$. We follow exactly the arguments of [6]. We know that $\operatorname{Dim}((z-1) M)=4$ and that any direct summand of $(z-1) M$ must have even dimension since it is free on restriction to $\langle\bar{x}\rangle$ for any $x$ in $P$. Consequently, there are at most two summands and the variety $V_{\bar{P}}((z-1) M)$ is the union of at most two lines. However, the variety does not contain any $\mathbb{F}_{2}$-rational line since such a line corresponds to a subgroup $\langle\bar{x}\rangle$ and we know that $\langle\bar{x}\rangle$ acts freely on the module. On the other hand, $(z-1) M$ is defined over $\mathbb{F}_{2}$ and hence its variety is $\mathbb{F}_{2}$-rational. The only possibility is that the variety is the zero set of a quadratic polynomial which is irreducible over $\mathbb{F}_{2}$. Over $k$, such a polynomial splits into two distinct linear factors. It follows that the module $(z-1) M$ is the direct sum of two submodules (by Theorem 2.7(8)), the variety of each being the zero set of one of the factors. Hence, this case is settled.

We now suppose that the order of $P$ is greater than 8 . We consider the exact sequence

$$
0 \rightarrow(z-1) M \rightarrow M_{0} \stackrel{\zeta}{\rightarrow} k \rightarrow 0
$$

where $\zeta$ is the natural quotient map. By Proposition 3.1, $M_{0} \cong \Omega^{2}\left(k_{\bar{P}}\right)$ and so $\zeta$ represents a cohomology element in

$$
\operatorname{Ext}_{k \bar{P}}^{2}(k, k) \cong \operatorname{Hom}_{k \bar{P}}\left(\Omega^{2}(k), k\right) .
$$

Hence, by Theorem 2.7(9), $V_{\bar{P}}((z-1) M)=V_{\bar{P}}(\zeta)$ is the zero locus of the cohomology element $\zeta$ (note that $(z-1) M=L_{\zeta}$ in the standard notation, used for instance in [6] and [14]).

Let $\bar{x}$ denote the central involution in the dihedral group $\bar{P}$. Because $\bar{x}$ acts freely on $(z-1) M$, the sequence splits on restriction to $\langle\bar{x}\rangle$ and it follows that the restriction of $\zeta$ to the cyclic center $\langle\bar{x}\rangle$ of $\bar{P}$ is not zero.

Now we follow the method of [6]. Because the element $\zeta$ restricts to a nonnilpotent element of the cohomology ring of the center of $\bar{P}, V_{\bar{P}}(\zeta)$ is the union of two nonempty 
closed sets which are in different components of the variety $V_{\bar{P}}(k)$. These components correspond to the two conjugacy classes of maximal elementary abelian subgroups of $\bar{P}$ and hence we get the decomposition of $(z-1) M$ into the direct sum of two submodules $N_{1}$ and $N_{2}$ having the properties stated in (4).

To prove the statement about dimensions, we note that

$$
\operatorname{Dim}\left(\Omega^{2}(k)\right)=|P|+1 \quad \text { so that } \operatorname{Dim}((z-1) M)=|P| / 2 .
$$

The two modules $N_{1}$ and $N_{2}$ must have the same dimension because there is an outer automorphism of $P$ of order 2 which fixes $z$, preserves the module $\Omega^{2}(k)$, and interchanges the two components of the variety. Hence it must interchange the modules $N_{1}$ and $N_{2}$. So $\operatorname{Dim}\left(N_{i}\right)=|P| / 4$ for $i=1,2$.

Finally we prove the indecomposability of $N_{1}$ and $N_{2}$. Let $H$ be a cyclic subgroup of $P$ of index 2 . Then $M \downarrow \stackrel{P}{H} \cong \Omega^{2}(k) \oplus$ (free) $=k \oplus$ (free) and therefore $((z-1) M) \downarrow \frac{P}{H}$ is a free $k \bar{H}$-module of dimension $|P| / 2=2|H|$. Thus $((z-1) M) \downarrow \frac{P}{H} \cong k \bar{H} \oplus k \bar{H}$, a direct sum of two indecomposable modules of dimension $|P| / 4$. This forces $N_{1}$ and $N_{2}$ to be indecomposable.

Remark 3.3. We have chosen for simplicity to work over an algebraically closed field $k$, but we note that if $P$ is quaternion of order 8 , then $(z-1) M$ decomposes as $N_{1} \oplus N_{2}$ whenever the base field $k$ contains cubic roots of unity, because the two lines in $V_{\bar{P}}((z-1) M)$ are not $\mathbb{F}_{2}$-rational but they are defined over $\mathbb{F}_{4}$. In contrast, if $P$ is quaternion of order at least 16 , then the two lines in $V_{\bar{P}}((z-1) M)$ are $\mathbb{F}_{2}$-rational and the decomposition $(z-1) M=N_{1} \oplus N_{2}$ holds over any field $k$ of characteristic 2 .

Now we pass from 2-groups to the general case.

Proposition 3.4. Let $G$ be a group with a quaternion Sylow 2-subgroup $P$ and assume that the unique involution $z$ of $P$ is central in $G$. Let $M=\Omega^{2}(k)$.

(1) The support variety $V_{\bar{G}}((z-1) M)$ has two components $V_{1}$ and $V_{2}$ and the $k \bar{G}$-module $(z-1) M$ decomposes as $(z-1) M \cong L_{1} \oplus L_{2}$, where $V_{\bar{G}}\left(L_{1}\right)=V_{1}$ and $V_{\bar{G}}\left(L_{2}\right)=V_{2}$.

(2) For $i=1,2$, we have $L_{i} \downarrow P \underset{P}{G} \cong N_{i} \oplus Q_{i}$, where $N_{i}$ is the $k \bar{P}$-module of Proposition 3.2 and $Q_{i}$ is a projective $k \bar{P}$-module.

(3) $\operatorname{Dim}\left(L_{i}\right)$ is congruent to $|P| / 4$ modulo $|P| / 2$.

(4) $L_{1}$ and $L_{2}$ are indecomposable.

Proof. We know that $M \downarrow{ }_{P}^{G} \cong \Omega^{2}\left(k_{P}\right) \oplus$ (proj) and consequently

$$
((z-1) M) \downarrow \frac{\bar{G}}{P}=(z-1)\left(M \downarrow{ }_{P}^{G}\right) \cong(z-1) \Omega^{2}\left(k_{P}\right) \oplus \text { (proj) }
$$

as $k \bar{P}$-modules. So by Proposition 3.2 , the support variety of the restriction $((z-1) M) \downarrow \frac{\bar{G}}{P}$ is the union of two components. We first note that these components are not conjugate under the action of $G$. If $|P|=8$, this is because the variety $V_{\bar{P}}((z-1) M)$ consists of two lines which cannot be conjugate under any automorphism of the quaternion group. Likewise, if $|P|>8$, the components of $V_{\bar{P}}((z-1) M)$ are not conjugate 
because there is no element of $\bar{G}$ which interchanges the two conjugacy classes of maximal elementary abelian subgroups of the dihedral group $\bar{P}$. Consequently, in either case, the support variety $V_{\bar{G}}((z-1) M)$ also has two components $V_{1}$ and $V_{2}$. Hence the $k \bar{G}$ module $(z-1) M$ must decompose as a direct sum

$$
(z-1) M=L_{1} \oplus L_{2}
$$

in such a way that $V_{\bar{G}}\left(L_{i}\right)=V_{i}$ for $i=1,2$ (see Theorem 2.7(8)).

By construction, we have $L_{i} \downarrow \frac{\bar{G}}{P}=N_{i} \oplus Q_{i}$ where $N_{i}$ is the module of Proposition 3.2 and $Q_{i}$ is a projective $k \bar{P}$-module. Since $\operatorname{Dim}\left(N_{i}\right)=|P| / 4$ and $\operatorname{Dim}\left(Q_{i}\right)$ is a multiple of $|P| / 2$, we deduce that $\operatorname{Dim}\left(L_{i}\right)$ is congruent to $|P| / 4$ modulo $|P| / 2$.

To prove the indecomposability of $L_{1}$ and $L_{2}$, we assume that $L_{i}=L_{i}^{\prime} \oplus L_{i}^{\prime \prime}$. Since $L_{i} \downarrow \frac{\bar{G}}{P}=N_{i} \oplus Q_{i}$ and $N_{i}$ is indecomposable, we get $L_{i}^{\prime} \downarrow \frac{\bar{G}}{P}=N_{i} \oplus Q_{i}^{\prime}$ and $L_{i}^{\prime \prime} \downarrow \frac{\bar{G}}{P}=Q_{i}^{\prime \prime}$, where $Q_{i}^{\prime}$ and $Q_{i}^{\prime \prime}$ are projective $k \bar{P}$-modules. Therefore $L_{i}^{\prime \prime}$ is projective, because $\bar{P}$ is a Sylow 2-subgroup of $\bar{G}$. By a result to be proved in the next section (see Proposition 4.2), this implies that the $k G$-module $M$ also has a projective direct summand $R$ such that $(z-1) R \cong L_{i}^{\prime \prime}$. But $M$ is indecomposable, so $R=0$, hence $L_{i}^{\prime \prime}=0$ and $L_{i}^{\prime}=L_{i}$.

Proposition 3.4 will be sufficient for the construction of exotic endotrivial modules and the determination of the group $T(G)$ of endotrivial modules in Section 4. However, for more specific information about endotrivial modules, we shall need the following result.

Proposition 3.5. Let $G$ be a group with a quaternion Sylow 2-subgroup P. Then we have the following.

(1) $\Omega^{4}(k) \cong k$.

(2) $\Omega^{2}(k)$ is self-dual.

(3) Assume that the unique involution $z$ of $P$ is central in $G$. Let $M=\Omega^{2}(k)$. Then the one-dimensional module $M_{0} /(z-1) M$ is the trivial module.

Proof. (1) The result is well-known, but we sketch an argument. Let $H=N_{G}(P)$. The Green correspondents of $\Omega^{4}\left(k_{G}\right)$ and $k_{G}$ are $\Omega^{4}\left(k_{H}\right)$ and $k_{H}$ respectively. So it suffices to prove the result over $H$. If $|P|>8$, then $\operatorname{Aut}(P)$ is a 2-group and therefore $N_{G}(P) / P C_{G}(P)=1$, that is, $H=P C_{G}(P)$. Then a complement $C$ of $P$ in $H$ centralizes $P$ and $H=P \times C$. Now $C$ acts trivially on $\Omega^{4}\left(k_{H}\right)$ and so this module is inflated from $\Omega^{4}\left(k_{P}\right)$. Finally it is well-known that for a quaternion group $P$, we have $\Omega^{4}\left(k_{P}\right) \cong k$ (see for instance [16, Proposition 3.16]). If $|P|=8$, then $N_{G}(P) / P C_{G}(P)$ has order 1 or 3. Again $P C_{G}(P)=P \times C$ and $C$ is normal in $H$. Since $C$ acts trivially on $\Omega^{4}\left(k_{H}\right)$, we are left with the group $H / C$ which is isomorphic to either $P$ or $P \rtimes C_{3}$. In the latter case, a direct computation (by hand as in [16] or using MAGMA [4]) shows that $\Omega^{4}(k) \cong k$.

(2) It follows from (1) that $\Omega^{2}(k) \cong \Omega^{-2}(k)$, that is, $\Omega^{2}(k) \cong \Omega^{2}(k)^{*}$.

(3) We know that $M_{0} /(z-1) M$ is one-dimensional (because $M \downarrow\langle z\rangle=k \oplus($ free)). Now one-dimensional modules are detected on restriction to $H=N_{G}(P)$. This follows either from the Green correspondence or from the fact that $H[G, G]=G$ (because $H[G, G]$ is 
normal in $G$ because it contains $[G, G]$ and selfnormalizing since it contains $H$ ). Therefore, it suffices to prove the result for $H$. As in part (1), $C$ acts trivially and it suffices to prove the result for $H / C$, which is isomorphic to either $P$ or $P \rtimes C_{3}$. In the former case any one-dimensional module is trivial, while in the latter case we conclude by a direct computation.

\section{Groups with quaternion Sylow 2-subgroup}

Let $G$ be a group with a quaternion Sylow 2-subgroup $P$. Our purpose in this section is to determine the group $T(G)$ of endotrivial modules for $G$. We continue to assume that $k$ is an algebraically closed field of characteristic 2. We let $z$ be the unique involution of $P$ and $H=C_{G}(z)$. Then $H$ is strongly 2 -embedded in $G$ and therefore we have the following result.

Lemma 4.1. The restriction map $\operatorname{Res}_{H}^{G}: T(G) \rightarrow T(H)$ is an isomorphism. Moreover, the Green correspondent of any indecomposable endotrivial $k H$-module is an indecomposable endotrivial $k G$-module.

Proof. The first statement is proved in [8, Proposition 2.8] or [22, Lemma 2.7]. The second statement is actually implicit in the first. The thing to notice here is that $H$ contains the normalizer of $P$ and if $g \in G, g \notin H$ then $P \cap g P g^{-1}=\{1\}$. Hence the Mackey formula tells us that if $M$ is an indecomposable $k H$-module, then $\left(M \uparrow{ }_{H}^{G}\right) \downarrow{ }_{H}^{G} \cong M \oplus$ (proj). This is the essence of the proof of the first statement of the lemma. The statement about Green correspondents is now obvious.

Thus it suffices to determine $T(H)$. Now $H$ has a nontrivial normal 2-subgroup $\langle z\rangle$. Therefore, by [22, Lemma 2.6], there is an exact sequence

$$
0 \rightarrow X(H) \rightarrow T(H) \stackrel{\operatorname{Res}_{P}^{H}}{\longrightarrow} T(P)
$$

where $X(H)$ denotes the subgroup of $T(H)$ consisting of the classes of all one-dimensional $k H$-modules. Clearly $X(H)$ is isomorphic to $\operatorname{Hom}\left(H, k^{*}\right)$, hence to the $2^{\prime}$-part of the abelianization of $H$.

We are going to prove that the restriction map $\operatorname{Res}_{P}^{H}$ is surjective and we do this by constructing exotic endotrivial modules for the group $H$. This is based on a construction which was already used in [13] and [6], and which takes the following form in characteristic 2. Note that, for $p$-groups, part (2) appears already in [11, Lemma 3.3] and [13, Lemma 5.3].

Proposition 4.2. Let $G$ be a group with a central involution $z$ and let $\bar{G}=G /\langle z\rangle$. Let $M$ be a $k G$-module such that $M \downarrow\langle z\rangle=k \oplus$ (proj). Assume that $L$ is a direct summand of $(z-1) M$.

(1) There exist submodules $\{0\} \subseteq V \subseteq U \subseteq M$ such that the subquotient $W=U / V$ has the properties that $W \downarrow \underset{\langle z\rangle}{G} \cong k \oplus$ (proj) and $(z-1) W \cong L$. 
(2) If $L$ is a projective $k \bar{G}$-module, then $W \cong K \oplus Q$ for some one-dimensional $k G$ module $K$ and some projective $k G$-module $Q$ such that $(z-1) Q \cong L$. Moreover, $Q$ is also isomorphic to a direct summand of $M$ (as a $k G$-module).

Proof. Set $(z-1) M=L \oplus L^{\prime}$. As in Section 3, we let $M_{0}=\{m \in M \mid(z-1) m=0\}$. Multiplication by $z-1$ induces an isomorphism from $M / M_{0}$ to $(z-1) M$ and $M$ has a filtration

$$
\{0\} \subset(z-1) M \subset M_{0} \subset M
$$

with the top $M / M_{0}$ isomorphic to the bottom $(z-1) M$ and with a one-dimensional middle module $M_{0} /(z-1) M$ (because $M \downarrow_{\langle z\rangle}^{G} \cong k \oplus$ (free)). Moreover, $M / M_{0}=N \oplus N^{\prime}$, with $N \cong L$ and $N^{\prime} \cong L^{\prime}$ via multiplication by $z-1$.

Let $U$ be the inverse image of $N$ in $M$, so that $U / M_{0}=N$ and $(z-1) U=L$. Let $W=U / L^{\prime}$. As before, let $W_{0}=\{w \in W \mid(z-1) w=0\}$. By construction we have isomorphisms of $k \bar{G}$-modules

$$
(z-1) W \cong L \text { and } W / W_{0} \cong N \cong L .
$$

In particular, the rank of multiplication by $z-1$ on $W$ is the dimension of $L$, and the dimension of $W$ is $2 \operatorname{Dim}(L)+1$. It follows that $W \downarrow_{\langle z\rangle}^{G}$ is the direct sum of a trivial module and a free module (because the rank of multiplication by $(z-1)$ is 0 on $k$ and is 1 on $k\langle z\rangle$, and these are the only indecomposable $k\langle z\rangle$-modules). This proves (1).

Assume now that $L$ is projective, so that $(z-1) W$ is a projective $k \bar{G}$-module. Then $(z-1) W$ is also an injective $k \bar{G}$-module, and the exact sequence

$$
0 \rightarrow(z-1) W \rightarrow W_{0} \rightarrow W_{0} /(z-1) W \rightarrow 0
$$

splits and $W_{0}$ has a one-dimensional submodule $K$ such that $W_{0}=(z-1) W \oplus K$. We claim that the $k G$-module $Q=W / K$ is projective. It suffices to prove this on restriction to a Sylow $p$-subgroup $P$ of $G$. Notice that $Q_{0}=(z-1) Q=(z+1) Q$ and this is isomorphic to $(z-1) W$, hence projective over $k \bar{P}$. Moreover $Q /(z+1) Q \cong(z+1) Q$ via multiplication by $z+1$. Now $\bar{P}$ is a $p$-group and $(z+1) Q$ is free over $k \bar{P}$. Therefore

$$
|\bar{P}| \cdot \operatorname{Dim}\left(\left(\sum_{x \in \bar{P}} x\right) \cdot(z+1) Q\right)=\operatorname{Dim}((z+1) Q) .
$$

Since $\sum_{u \in P} u=\left(\sum_{x \in \bar{P}} x\right)(z+1)$, it follows that

$|P| \operatorname{Dim}\left(\left(\sum_{u \in P} u\right) \cdot Q\right)=2|\bar{P}| \operatorname{Dim}\left(\left(\sum_{x \in \bar{P}} x\right) \cdot(z+1) Q\right)=2 \operatorname{Dim}((z+1) Q)=\operatorname{Dim}(Q)$.

This implies that $Q$ is a free $k P$-module, because $P$ is a $p$-group and $\sum_{u \in P} u$ annihilates every nonprojective indecomposable $k P$-module.

Now the projectivity of $Q$ implies that $W \cong K \oplus Q$. Because $W$ is a subquotient of $M$ and $Q$ is an injective module, $Q$ must be isomorphic to a direct summand of $M$ (as a $k G$-module). 
Using the construction of Proposition 4.2 we now show the existence of exotic endotrivial modules. Later in this section (in the proof of Theorem 4.5), we see that, with some additional argument, the hypothesis on the centrality of $z$ can be removed from the next theorem.

Theorem 4.3. Let $G$ be a group with a quaternion Sylow 2-subgroup P. Assume that the unique involution $z \in P$ is central in $G$. Then we have the following.

(1) There exist exotic endotrivial $k G$-modules, that is, indecomposable endotrivial $k G$ modules whose dimension is congruent to $|P| / 2+1$ modulo $|P|$.

(2) More precisely, for $i=1,2$, there exists an exotic endotrivial $k G$-module $W_{i}$ such that $(z-1) W_{i} \cong L_{i}$ (as $k \bar{G}$-modules), where $L_{1}$ and $L_{2}$ are the two $k \bar{G}$-modules constructed in Proposition 3.4.

Proof. Let $M=\Omega^{2}(k)$ and note that

$$
M \downarrow \stackrel{G}{G(z\rangle} \cong \Omega^{2}\left(k_{\langle z\rangle}\right) \oplus(\text { proj }) \cong k \oplus(\text { proj }) .
$$

Recall that $(z-1) M \cong L_{1} \oplus L_{2}$, as in Proposition 3.4, and apply Proposition 4.2 to $L_{i}$ for $i=1,2$. There exists a $k G$-module $W_{i}$ such that $W_{i} \downarrow_{\langle z\rangle}^{G} \cong k \oplus(\operatorname{proj})$ and $(z-1) W_{i} \cong L_{i}$. The restriction $W_{i} \downarrow_{\langle z\rangle}^{G}$ is an endotrivial module, because it is the direct sum of a trivial module and a free module. Since $\langle z\rangle$ is the only nontrivial elementary abelian 2-subgroup of $G$, it must be the case that $W_{i}$ is an endotrivial module (by Lemma 2.2). If $W_{i}$ had a nontrivial projective direct summand, then $(z-1) W_{i} \cong L_{i}$ would have a nontrivial projective direct summand as a $k \bar{G}$-module, hence $(z-1) M$ too. But then $M=\Omega^{2}(k)$ itself would have a nontrivial projective direct summand, by Proposition 4.2. This is impossible and it follows that $W_{i}$ is indecomposable. Finally, since $\operatorname{Dim}\left(L_{i}\right)$ is congruent to $|P| / 4$ modulo $|P| / 2$ (see Proposition 3.4) and since $\operatorname{Dim}\left(W_{i}\right)=2 \operatorname{Dim}\left(L_{i}\right)+1$, we see that $\operatorname{Dim}\left(W_{i}\right)$ is congruent to $|P| / 2+1$ modulo $|P|$. Hence $W_{i}$ is exotic.

The proof provides two nonisomorphic exotic $k G$-modules $W_{1}$ and $W_{2}$, but note that there are many other possible exotic modules, because if $W$ is exotic and $A$ is one-dimensional, then obviously $A \otimes W$ is again exotic.

Remark 4.4. By Remark 3.3, one needs cubic roots of unity for the existence of exotic endotrivial modules in the case where $P$ has order 8 .

The preceding discussion now implies the following theorem.

Theorem 4.5. Suppose that $G$ is a finite group with a quaternion Sylow 2-subgroup $P$ and let $H=C_{G}(z)$, where $z$ is the unique involution of $P$.

(1) The restriction map $\operatorname{Res}_{P}^{G}: T(G) \rightarrow T(P)$ is surjective.

(2) Let $\hat{X}(G)$ denote the subgroup of $T(G)$ generated by the classes of the Green correspondents of the 1-dimensional $k H$-modules. Then we have a split short exact sequence

$$
0 \rightarrow \hat{X}(G) \rightarrow T(G) \stackrel{\operatorname{Res}_{P}^{G}}{\longrightarrow} T(P) \rightarrow 0 .
$$

(3) $\hat{X}(G) \cong X(H)$. 
Proof. First suppose that $z$ is central in $G$. By Theorem 2.4, the group $T(P)$ is generated by the classes of $\Omega\left(k_{P}\right)$ and of an exotic endotrivial $k P$-module $U$. Clearly, the class of $\Omega\left(k_{G}\right)$ restricts to the class of $\Omega\left(k_{P}\right)$. An exotic endotrivial $k G$-module $W$ (which exists by Theorem 4.3) restricts to an endotrivial $k P$-module whose dimension is congruent to $|P| / 2+1$ modulo $|P|$. The unique indecomposable nonprojective (and endotrivial) summand $\left(W \downarrow \downarrow_{P}^{G}\right)_{\diamond}$ of $W \downarrow \stackrel{G}{G}$ must also have dimension congruent to $|P| / 2+1$ modulo $|P|$ and is therefore exotic. It follows that $(W \downarrow \stackrel{G}{G})_{\diamond}$ is isomorphic to one of the two exotic $k P$ modules $U$ or $\Omega^{2}(U)$ (and actually $\operatorname{Dim}\left(\left(W \downarrow{ }_{P}^{G}\right)_{\diamond}\right)=|P| / 2+1$ by Remark 2.6). As a consequence, the image of the restriction map $T(G) \rightarrow T(P)$ includes a set of generators of $T(P)$ and hence the map is surjective in this case.

For the general case, when $z$ is not central, we provide two different proofs. Each has its own interest and advantages. Let $H=C_{G}(z)$ and let $W$ be an exotic endotrivial $k H$ module. By Lemma 4.1, there is an indecomposable endotrivial $k G$-module $V$ such that $V \downarrow_{H}^{G} \cong W \oplus$ (proj). Then $\operatorname{Dim}(V)$ is congruent to $\operatorname{Dim}(W)$ modulo $|P|$, so $V$ is exotic. For the other proof, we use the Brauer-Suzuki Theorem [5] which tells us that $G / O_{2^{\prime}}(G)$ has a central involution. In addition, $O_{2^{\prime}}(G)$ acts trivially on $\Omega^{2}(k)$ and on every module in the principal block. Therefore our constructions show that there is an exotic endotrivial $k\left[G / O_{2^{\prime}}(G)\right]$-module, which can be viewed as a $k G$-module by inflation. This is still endotrivial, as we see from the definition and the fact that the inflation of a projective module remains projective. This proves part (1).

From (1), we have an exact sequence

$$
0 \rightarrow X(H) \rightarrow T(H) \stackrel{\operatorname{Res}_{P}^{H}}{\longrightarrow} T(P) \rightarrow 0
$$

which splits because $T(P)$ is a 2-group (of order 8) and $X(H)$ has odd order. Using the isomorphism of Lemma 4.1, we obtain $T(G) \cong T(H) \cong \hat{X}(G) \oplus T(P)$. That is, $\hat{X}(G)$ is the inverse image of $X(H)$ under the restriction isomorphism. This proves (2) and (3).

In Theorem 4.5, the splitting of the exact sequence exists and is unique. Our next goal is to give an explicit description of this splitting, by using the additional information provided by Proposition 3.5. The group $T(P)$ is generated by the classes of $\Omega\left(k_{P}\right)$ and $U$, where $U$ is an exotic $k P$-module. The splitting of $\operatorname{Res}_{P}^{H}: T(H) \rightarrow T(P)$ must lift each of these two generators to an element of $T(H)$ of the same order.

Proposition 4.6. The image of the unique splitting of $\operatorname{Res}_{P}^{H}: T(H) \rightarrow T(P)$ is generated by the classes of $\Omega\left(k_{H}\right)$ (of order 4 ) and one of the two modules $W_{1}, W_{2}$ constructed in Theorem 4.3 (of order 2 , that is, self-dual). Moreover, $\Omega^{2}\left(W_{1}\right) \cong W_{2}$.

Proof. It is clear that $\Omega\left(k_{P}\right)$ lifts to $\Omega\left(k_{H}\right)$, and this still has order 4 by Proposition 3.5 . Now each of the two exotic $k P$-modules is self-dual (that is, its class in $T(P)$ has order 2). We know that the two exotic $k H$-modules $W_{1}$ and $W_{2}$ constructed in Theorem 4.3 restrict to the two exotic $k P$-modules. So we only have to prove that $W$ is self-dual, where $W=W_{1}$ or $W=W_{2}$. If $\operatorname{Res}_{P}^{H}[W]=[U]$, then both $[W]$ and its dual $\left[W^{*}\right]$ restrict to $[U]$, because $U$ is self-dual. Since the kernel of $\operatorname{Res}_{P}^{H}$ is the group of linear characters of $H$, 
there is a one-dimensional $k H$-module $A$ with the property that $W^{*} \cong A \otimes W$. Tracking the construction of $W$, we note that $W$ has a filtration with three successive quotients

$$
(\bar{W}, K, \bar{W})
$$

where $\bar{W}=(z-1) W$ and the middle module $K=W_{0} /(z-1) W$ is one-dimensional. Now the construction of $W$ comes from the $k H$-module $M=\Omega^{2}\left(k_{H}\right)$ which has a similar filtration. The construction of $W$ shows that its middle module $K$ is isomorphic to the middle one-dimensional module $M_{0} /(z-1) M$ (see the proof of Proposition 4.2). But $M_{0} /(z-1) M$ is the trivial module by Proposition 3.5 , so $K$ is the trivial module. It follows that $A \otimes W$ has a filtration with three successive quotients

$$
(A \otimes \bar{W}, A, A \otimes \bar{W}) .
$$

On the other hand, $W^{*}$ has a filtration with three successive quotients

$$
\left(\bar{W}^{*}, K, \bar{W}^{*}\right) \text {. }
$$

This implies that $A \cong K$ is the trivial module, so $W^{*} \cong A \otimes W \cong W$.

To prove that $\Omega^{2}\left(W_{1}\right) \cong W_{2}$, we observe that, since the order of $X(H)$ is odd, there are exactly three elements of order 2 in $T(H)$, namely the classes of $\Omega^{2}(k), W_{1}$ and $W_{2}$. This forces the equality $\left[\Omega^{2}(k)\right]+\left[W_{1}\right]=\left[W_{2}\right]$ in $T(H)$, that is, $\left[\Omega^{2}\left(W_{1}\right)\right]=$ $\left[\Omega^{2}(k) \otimes W_{1}\right]=\left[W_{2}\right]$. Since both $k H$-modules $\Omega^{2}\left(W_{1}\right)$ and $W_{2}$ are indecomposable, they must be isomorphic.

\section{Uniserial endotrivial modules}

In this section we prove the existence of uniserial endotrivial modules of dimension congruent to $\pm 1(\bmod |P| / 2)$ for any finite group $G$ with a quaternion Sylow 2-subgroup $P$. More precisely, if $W_{1}, W_{2}$ are the two exotic modules constructed in Theorem 4.3, then either $W_{i}$ is uniserial or $\Omega\left(W_{i}\right)$ is uniserial (or both) for $i=1,2$. Recall that $W_{2} \cong \Omega^{2}\left(W_{1}\right)$. The method is a direct application of the techniques used in Proposition 3.4 and Theorem 4.3, alongside an inspection of the results in [20] in the six cases where the given basic algebra may arise as the principal block of a group algebra. We use Erdmann's notation of [20, pp. 303-305] and also the usual diagrammatic representations of modules (see e.g. [18]). As before, if we say that a module has composition factors $(A, B, C, \ldots)$, we read these from head to socle of the module. Now, recall that the diagram for $\Omega^{2}(k)$ has the form

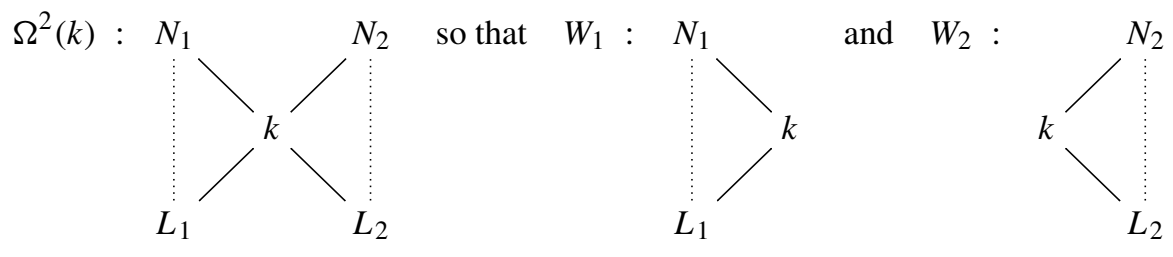


where $N_{i} \cong L_{i}$ is uniserial for $i=1,2$. An edge is dotted to mean that there may or may not be a nontrivial extension between the modules. This determines which of the modules $W_{1}, W_{2}$, or their respective syzygies is uniserial. Indeed, one fact that follows from the explicit computations is that if an exotic module is not uniserial, then its syzygy is uniserial. We also remark that if an exotic module is uniserial, then its syzygy may also be uniserial.

The principal 2-block of a group with quaternion Sylow 2-subgroup can have one, two or three simple modules. We analyze these cases by subcases according to the results of [20].

\subsection{One simple module}

The simple module must be the trivial module $k$. In particular, this situation occurs when $G=P$ is quaternion. In this case, the construction shows that any exotic endotrivial module $W_{i}$ is not uniserial and has composition length $|P| / 2+1$, whereas its syzygy $\Omega\left(W_{i}\right)$ is uniserial and has composition length $|P| / 2-1$ for $i=1,2$. In addition, the modules $N_{i}$ are both uniserial of composition length $|P| / 4$ for $i=1,2$.

\subsection{Two simple modules}

There are two possibilities. Write $k$ and $S$ for the two nonisomorphic simple modules. Note that both are self-dual. According to [17, (6.8)], such an algebra occurs as principal block of a finite group $G$ having a subgroup isomorphic to $\mathrm{SL}_{2}(q)$ of index 2. The case (i) is when $q \equiv 1(\bmod 4)$ and (ii) when $q \equiv 3(\bmod 4)$.

(i) For the type $Q(2 \mathcal{A})$, exactly one exotic module $W$ is uniserial of length 3, with composition factors $(S, k, S)$, and $\Omega^{3}(W)$ is also uniserial. Indeed, we have $N_{1}=S$ and $N_{2}$ has length $3|P| / 8$, with composition factors $(k, S, k, k, S, k, \ldots, S, k)$. Moreover, there is a nontrivial extension between the socle of $N_{2}$ and the head of $L_{2}$, implying that $\Omega^{2}(W)$ is not uniserial. We conclude that $\Omega^{3}(W)$ is uniserial with composition factors $(S, k, k, S, k, \ldots, k, S, k)$ and length $3|P| / 4-1$.

(ii) For the type $Q(2 \mathcal{B})_{1}$, no exotic module is uniserial. Both syzygies of the exotic modules are uniserial of length $3|P| / 4-1$, with composition factors $(k, S, S, k, S, S, \ldots, k, S)$ and $(S, k, S, S, k, S, \ldots, S, k)$. Explicitly, we find that the modules $N_{i}$ have composition length $|P| / 4$ and $3|P| / 8$ with composition factors $(S, S, \ldots, S)$ and $(k, S, k, k, S, k, \ldots, S, k)$.

\subsection{Three simple modules}

There are three possibilities, giving rise to the well known examples. Write $k, S, T$ for the three simple modules.

(i) For the type $Q(3 \mathcal{A})_{2}$, which occurs as the principal block of $\operatorname{SL}_{2}(q)$ for $q \equiv 1$ $(\bmod 4)$, both exotic modules are uniserial of length 3 , with composition factors $(S, k, S)$ and $(T, k, T)$. In particular, the modules $N_{i}$ are simple and all the simple modules are self-dual. 
(ii) For the type $Q(3 \mathcal{K})$, which occurs as the principal block of $\mathrm{SL}_{2}(q)$ for $q \equiv 3$ $(\bmod 4)$, none of the exotic modules is uniserial. Instead, their syzygies are uniserial and both have length 3 , with composition factors $(S, k, S)$ and $(T, k, T)$. Moreover, the modules $N_{i}$ have length $|P| / 4$ and composition factors $(S, T, \ldots, S, T)$ and $(T, S, \ldots, T, S)$. In this case $S^{*} \cong T$.

(iii) For the type $Q(3 \mathcal{B})$, which occurs as the principal block of the double cover of $A_{7}$ (with $|P|=16$ ), one exotic endotrivial module is uniserial of length 3 and composition factors $(S, k, S)$, whereas the other exotic endotrivial module is not uniserial, but its syzygy is and has composition factors $(S, k, T, k, S, k, T)$. We observe that in this situation, each exotic module and its syzygy has composition length independent of the size of $P$.

We end with a remark on the dimensions of the uniserial endotrivial modules in the case of the groups $\mathrm{SL}_{2}(q)$. It is known that the two nontrivial simple modules have dimension $(q-1) / 2$. Since each uniserial endotrivial module in (i) and (ii) has composition series $(S, k, S)$, where $S$ is a nontrivial simple module, we have the following.

Proposition 5.1. Let $G=\mathrm{SL}_{2}(q)$, with $q$ an odd prime power. Write $P$ for a Sylow 2-subgroup of $G$. Then there exist two nonisomorphic uniserial endotrivial modules of dimension $q$ and length 3. More precisely, these modules are exotic (and self-dual) if and only if $q \equiv 1(\bmod 4)$. For $q \equiv 3(\bmod 4)$, their syzygies are the two exotic modules and have dimension $1+(q-1)|P| / 8$.

\section{Groups with semi-dihedral Sylow 2-subgroup}

Suppose that $G$ is a finite group with a semi-dihedral Sylow 2-subgroup $P$. Our main aim in this section is to show that the restriction map $\operatorname{Res}_{P}^{G}: T(G) \rightarrow T(P)$ is split surjective. We continue to assume that $k$ is an algebraically closed field of characteristic 2 . We first discuss the general structure of $T(G)$.

Proposition 6.1. Let $G$ be a finite group with a semi-dihedral Sylow 2-subgroup P. Write $K(G)$ for the kernel of the restriction map $\operatorname{Res}_{P}^{G}: T(G) \rightarrow T(P)$.

(1) $K(G)$ is a finite group of odd order, isomorphic to a subgroup of the group $X(N)$ of one-dimensional representations of $N$, where $N=N_{G}(P)$.

(2) $T(G) \cong K(G) \oplus \operatorname{Im}\left(\operatorname{Res}_{P}^{G}\right)$.

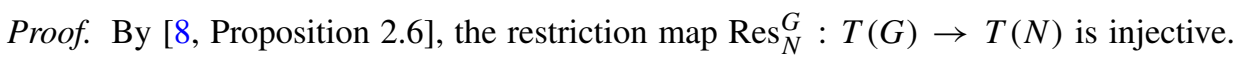
Now $N$ has a nontrivial normal 2-subgroup and therefore, by [22, Lemma 2.6], there is an exact sequence

$$
0 \rightarrow X(N) \rightarrow T(N) \stackrel{\operatorname{Res}_{P}^{N}}{\longrightarrow} T(P)
$$

where $X(N)$ denotes the subgroup of $T(N)$ consisting of the classes of all one-dimensional $k N$-modules. Clearly $X(N)$ is isomorphic to $\operatorname{Hom}\left(N, k^{*}\right)$, and hence it is also isomorphic to the $2^{\prime}$-part of the abelianization of $N$. It follows that $K(G)$ is the inverse 
image under $\operatorname{Res}_{N}^{G}$ of the odd order group $X(N)$. Since $T(P) \cong \mathbb{Z} / 2 \mathbb{Z} \oplus \mathbb{Z}$, the map $\operatorname{Res}_{P}^{G}$ splits and $T(G) \cong K(G) \oplus \operatorname{Im}\left(\operatorname{Res}_{P}^{G}\right)$.

So we are left with the question of the surjectivity of $\operatorname{Res}_{P}^{G}: T(G) \rightarrow T(P)$. We use in an essential way the stable Auslander-Reiten quiver of $k G$, in particular the work of Webb [24] and Erdmann [20, 18, 19]. For any subgroup $S$ of $G$, we denote by $\Delta^{S}$ the component of the stable Auslander-Reiten quiver of $k S$ containing the module $\Omega\left(k_{S}\right)$. An AR-sequence stands for an Auslander-Reiten sequence (or almost split sequence). We first state the results we need.

Proposition 6.2. Let $G$ be a group with a semi-dihedral Sylow 2-subgroup $P$ and let $N=N_{G}(P)$.

(1) The Green correspondence induces an isomorphism $\Delta^{G} \cong \Delta^{N}$.

(2) $N \cong P \times X$ where $X$ is a group of odd order. Restriction induces an isomorphism $\Delta^{N} \cong \Delta^{P}$, with inverse induced by inflation from $P \cong N / X$ to $N$.

(3) $\Delta^{P}$ is a component of type $\mathbb{Z} D_{\infty}$ and $\Omega^{-1}\left(k_{P}\right)$ lies at the end of $\Delta^{P}$, with one predecessor.

(4) Let $R_{k}$ be the projective cover of the trivial module $k$ and let the heart of $R_{k}$ be the module $H_{k}=\operatorname{Rad}\left(R_{k}\right) / \operatorname{Soc}\left(R_{k}\right)$. There is an AR-sequence

$$
\mathcal{S}: \quad 0 \rightarrow \Omega(k) \rightarrow H_{k} \oplus R_{k} \rightarrow \Omega^{-1}(k) \rightarrow 0 .
$$

(5) All modules in $\Delta^{G}$ have $P$ as a vertex.

(6) Any AR-sequence terminating in a module in $\Delta^{G}$ splits on restriction to a proper subgroup of its vertex $P$.

Proof. (1) This is proved in Theorem D of [24].

(2) It is well-known that $\operatorname{Aut}(P)$ is a 2-group. Actually the proof given in [21, Lemma 7.7.2(vi)] for the automorphism group of a dihedral 2-group carries over verbatim for a semi-dihedral 2-group. It follows that $N=P C_{G}(P)$ and therefore $N=P \times X$, where $X$ is a group of odd order. Thus $X$ is in the kernel of the principal block of $k N$ and acts trivially on all modules in $\Delta^{N}$. Therefore restriction induces an isomorphism $\Delta^{N} \cong \Delta^{P}$ and inflation induces the inverse isomorphism.

(3) Shifting by the Heller translate $\Omega$ induces an isomorphism between $\Delta^{P}$ and the component of the stable Auslander-Reiten quiver of $k P$ containing the trivial module $k$. By [18, Lemma 7.1] or [20, Proposition II.10.1]), the latter is of type $\mathbb{Z} D_{\infty}$ and $k$ lies at the end of the component, with one predecessor.

(4) This is well-known (e.g. [24, Section 4] or [2, Proposition 4.12.7]).

(5) This is Theorem $C$ of [24].

(6) This is well-known (e.g. [24, Lemma 3.1] or [2, Proposition 4.12.10]).

The presence of a tree class $D_{\infty}$ is an exceptional case which only occurs in the semidihedral situation. (Note that this case was missing in [24, Proposition 5.6].) Moreover, Proposition 6.2 has the following consequence, already used in $[18,(2.3)]$ and in $[24$, Proposition 5.6]. 
Corollary 6.3. Let $G$ be a group with a semi-dihedral Sylow 2-subgroup $P$. Let $R_{k}$ be the projective cover of the trivial module $k$ and let $H_{k}=\operatorname{Rad}\left(R_{k}\right) / \operatorname{Soc}\left(R_{k}\right)$ be the heart of $R_{k}$. Then there is an AR-sequence of the form

$$
\mathcal{E}: \quad 0 \rightarrow V \rightarrow H_{k} \oplus R \rightarrow U \rightarrow 0
$$

where $V$ and $U$ are indecomposable modules in $\Delta^{G}, U$ is not isomorphic to $\Omega^{-1}(k)$, and $R$ is a projective $k G$-module. Moreover $V^{*} \cong U$.

Proof. By Webb's theorem [24, Theorem E], the heart $H_{k}$ of $R_{k}$ is indecomposable. In view of the AR-sequence $\mathcal{S}$, we see that $H_{k}$ is the only predecessor of $\Omega^{-1}(k)$ in $\Delta^{G}$. Since $\Delta^{G}$ is of type $\mathbb{Z} D_{\infty}$ by Proposition $6.2, H_{k}$ must appear in the middle of another AR-sequence of the form $\mathcal{E}$, where $U$ is not isomorphic to $\Omega^{-1}(k)$ and $R$ is some projective module.

Since $H_{k}$ is self-dual, the dual of $\mathcal{E}$ is an AR-sequence with $H_{k}$ in the middle. Since the other AR-sequence $\mathcal{S}$ is self-dual, the dual of $\mathcal{E}$ must be isomorphic to itself and hence $V^{*} \cong U$.

Corollary 6.3 is sufficient for our construction of exotic endotrivial modules, but we shall see at the end that much more can be proved about the AR-sequence $\mathcal{E}$. The main fact is that the modules $V$ and $U$ in Corollary 6.3 are endotrivial and this was first noticed by Bessenrodt [3]. More precisely, the use of Corollary 6.3 in the analysis of endotrivial modules is as follows.

Theorem 6.4. Let $G$ be a group with a semi-dihedral Sylow 2-subgroup P.

(1) The modules $V$ and $U$ in Corollary 6.3 are endotrivial.

(2) The endotrivial module $\Omega(U)$ is exotic and self-dual. In other words, its class $[\Omega(U)]$ has order 2 in $T(G)$.

(3) The restriction map $\operatorname{Res}_{P}^{G}: T(G) \rightarrow T(P)$ is surjective and split.

Proof. Consider the AR-sequence

$$
\mathcal{S}: \quad 0 \rightarrow \Omega(k) \rightarrow H_{k} \oplus R_{k} \rightarrow \Omega^{-1}(k) \rightarrow 0
$$

of Proposition 6.2. Let $Q$ be the unique maximal dihedral subgroup of $P$. Observe that $Q$ contains all the elements of order 2 in $P$. As a consequence, a $k G$-module is projective if and only if it is projective on restriction to $Q$ (see Theorem 2.7(2)). By Proposition 6.2, the above sequence $\mathcal{S}$ splits on restriction to $Q$. Since $\Omega(k) \downarrow \stackrel{G}{G} \cong \Omega\left(k_{Q}\right) \oplus$ (proj) and similarly for $\Omega^{-1}(k)$, we deduce that

$$
H_{k} \downarrow_{Q}^{G} \cong \Omega\left(k_{Q}\right) \oplus \Omega^{-1}\left(k_{Q}\right) \oplus(\text { proj }) .
$$

Now the other AR-sequence

$$
\mathcal{E}: \quad 0 \rightarrow V \rightarrow H_{k} \oplus R \rightarrow U \rightarrow 0
$$

of Corollary 6.3 also splits on restriction to $Q$, again by Proposition 6.2, using the fact that all the modules in the AR-component $\Delta^{G}$ have vertex $P$. Thus we obtain a sequence

$$
\mathcal{E} \downarrow_{Q}^{G}: \quad 0 \rightarrow V \downarrow_{Q}^{G} \rightarrow \Omega\left(k_{Q}\right) \oplus \Omega^{-1}\left(k_{Q}\right) \oplus(\text { proj }) \rightarrow U \downarrow \stackrel{G}{Q} \rightarrow 0 .
$$


Now $V \downarrow \stackrel{G}{Q}$ cannot be projective, otherwise $V$ would be projective, and similarly for $U \downarrow \stackrel{G}{Q}$. Consequently, $U$ and $V$ have the property that

$$
U \downarrow_{Q}^{G} \cong \Omega^{\varepsilon}\left(k_{Q}\right) \oplus(\text { proj }) \quad \text { and } \quad V \downarrow_{Q}^{G} \cong \Omega^{-\varepsilon}\left(k_{Q}\right) \oplus(\text { proj })
$$

for $\varepsilon= \pm 1$ and for some projective modules. Note that, since $Q$ contains all the elements of order 2 in $P$, the restrictions of $U$ and $V$ to any elementary abelian 2-subgroup are endotrivial modules. Therefore $U$ and $V$ are endotrivial modules by Lemma 2.2, proving (1).

(2) Since $\mathcal{E}$ is an AR-sequence, $V \cong \Omega^{2}(U)$. On the other hand $V \cong U^{*}$ by Corollary 6.3. Therefore

$$
\Omega(U)^{*} \cong \Omega^{-1}\left(U^{*}\right) \cong \Omega^{-1}(V) \cong \Omega^{-1}\left(\Omega^{2}(U)\right) \cong \Omega(U)
$$

It follows that the class $[\Omega(U)]$ has order 2 in $T(G)$ (for it cannot be of order 1 because $\left.U \supsetneqq \Omega^{-1}(k)\right)$. Since the kernel $K(G)$ of $\operatorname{Res}_{P}^{G}$ has odd order by Proposition 6.1, $\operatorname{Res}_{P}^{G}([\Omega(U)])$ has order 2 in $T(P) \cong \mathbb{Z} / 2 \mathbb{Z} \oplus \mathbb{Z}$. Thus $\operatorname{Res}_{P}^{G}([\Omega(U)])$ is the class of the unique indecomposable endotrivial $k P$-module that is both self-dual and exotic. So the dimension of $\Omega(U)$ is congruent to $|P| / 2+1$ modulo $|P|$, and we conclude that $\Omega(U)$ must be exotic.

(3) By (2), the summand $\mathbb{Z} / 2 \mathbb{Z}$ of $T(P) \cong \mathbb{Z} / 2 \mathbb{Z} \oplus \mathbb{Z}$ is in the image of $\operatorname{Res}_{P}^{G}$. Clearly $\operatorname{Res}_{P}^{G}\left[\Omega\left(k_{G}\right)\right]=\left[\Omega\left(k_{P}\right)\right]$, which generates the summand $\mathbb{Z}$. Thus $\operatorname{Res}_{P}^{G}$ is surjective. There is an obvious splitting whose image is generated by $\left[\Omega\left(k_{G}\right)\right]$ and $[\Omega(U)]$.

Corollary 6.5. Let $G$ be a finite group with a semi-dihedral Sylow 2-subgroup P. Write $K(G)$ for the kernel of the restriction map $\operatorname{Res}_{P}^{G}: T(G) \rightarrow T(P)$. Then $T(G) \cong K(G) \oplus$ $T(P)$. In particular, if $P$ is selfnormalizing, then $T(G) \cong T(P)$.

The only difference with the quaternion case, which prevents us from concluding as before with the identification of $K(G)$, is that the centralizer in $G$ of the central involution $z$ of $P$ is not strongly 2 -embedded in general. So the restriction map $\operatorname{Res}_{C_{G}(z)}^{G}$ : $T(G) \rightarrow T\left(C_{G}(z)\right)$ may not be an isomorphism (even though it is injective by [8, Proposition 2.6]).

As announced before, we conclude with some additional information about the ARsequence $\mathcal{E}$ of Corollary 6.3. Most of the hard work for the proof has been done by K. Erdmann [20, 18, 19].

Proposition 6.6. Let $G$ be a group with a semi-dihedral Sylow 2-subgroup P. In the ARsequence $\mathcal{E}$ of Corollary 6.3, the projective module $R$ is zero. Moreover $V$ and $U$ are uniserial modules.

Proof. By [1, Proposition 4.11], an AR-sequence with a nonzero projective summand $R$ in the middle must be the standard AR-sequence ending in $\Omega^{-1}(T)$, where $T$ is a simple module. So if $R \neq 0$, we must have $R=R_{T}$ where $R_{T}$ is the projective cover of $T$ and the heart $H_{T}=\operatorname{Rad}\left(R_{T}\right) / \operatorname{Soc}\left(R_{T}\right)$ is isomorphic to $H_{k}$. Note that $T$ is nontrivial because the sequence $\mathcal{E}$ is not isomorphic to the sequence $\mathcal{S}$. 
Now we claim that there is no nontrivial simple module $T$ such that $H_{T} \cong H_{k}$. This follows from an inspection of Erdmann's lists in [18, 19]. By Olsson's results [23], the principal block of the group algebra $k G$ has either one, two, or three simple modules. There is nothing to prove if there is only one simple module. If there are two, then the family $\mathrm{V}$ does not occur as a block of a group algebra (by [18, Lemma 8.16]) and we see that in families I-IV the hearts of the two projective modules are not isomorphic. Similarly, if there are three simple modules, then the family VII does not occur as a block of a group algebra (by [19, Lemma 11.14]) and we see that in all the other families the hearts of any two projective modules are not isomorphic.

It follows now that $R=0$. The fact that $V$ and $U$ are uniserial follows from a direct inspection of the hearts, for all self-dual projective modules appearing in Erdmann's lists.

Remark 6.7. As noted already in [20, 19], an algebra of semi-dihedral type with three simple modules may occur as block algebra if it belongs to any family of type I-IV, whereas V-VIII are known to be non-examples of blocks. In Erdmann's paper, case IX is left open. Following a private communication with K. Erdmann, it turns out that an algebra in family IX is not a block algebra. Indeed, in the notation of [19], we have $n \geq 4$, and since $P_{1}$ is the unique self-dual indecomposable projective module in the list, we would have $S_{1}=k$. This would give an AR-sequence $\mathcal{E}$ with $V$ and $U$ uniserial. By taking the dual of the sequence, we get $U^{*} \cong V$. So the other two simple modules $S_{0}$ and $S_{2}$ must be dual to each other. Counting the multiplicities of each simple in $V$ and in $U$ yields the equation $s=k$ with $s=2$ and $k=2^{n-2}$, which is impossible for any $n \geq 4$.

Acknowledgments. The authors are sincerely grateful to K. Erdmann for several enlightening discussions, which have been considerably helpful for the last section of this paper.

Research of J. F. Carlson was partially supported by a grant from NSF.

\section{References}

[1] Auslander, M., Reiten, I.: Representation theory of Artin algebras IV. Invariants given by almost split sequences. Comm. Algebra 5, 443-518 (1977) Zbl 0396.16007 MR 0439881

[2] Benson, D. J.: Representation and Cohomology I, II. Cambridge Stud. Adv. Math. 30, 31, Cambridge Univ. Press (1991) Zbl 0718.20001(Vol. I), Zbl 0731.20001(Vol. II) MR 1110581(Vol. I), MR 1156302(Vol. II)

[3] Bessenrodt, C.: Endotrivial modules and the Auslander-Reiten quiver. In: Representation Theory of Finite Groups and Finite-Dimensional Algebras, Progr. Math. 95, Birkhäuser, Basel, 317-326 (1991) Zbl 0774.20006 MR 1112166

[4] Bosma, W., Cannon, J.: Handbook on Magma Functions. Sydney Univ. (1996)

[5] Brauer, R., Suzuki, M.: On finite groups of even order whose 2-Sylow group is a quaternion group. Proc. Nat. Acad. Sci. U.S.A. 45, 1757-1759 (1959) Zbl 0090.01901 MR 0109846

[6] Carlson, J.: Constructing endotrivial modules. J. Pure Appl. Algebra 206, 83-110 (2006) Zbl 1098.20004 MR 2220083

[7] Carlson, J., Hemmer, D., Mazza, N.: The group of endotrivial modules for the symmetric and alternating groups. Proc. Edinburgh Math. Soc. 53, 83-95 (2010) Zbl 1217.20004 MR 2579680 
[8] Carlson, J., Mazza, N., Nakano, D.: Endotrivial modules for finite groups of Lie type. J. Reine Angew. Math. 595, 93-119 (2006) Zbl 1106.20007 MR 2244799

[9] Carlson, J., Mazza, N., Nakano, D.: Endotrivial modules for the symmetric and alternating groups. Proc. Edinburgh Math. Soc. 52, 45-66 (2009) Zbl 1165.20005 MR 2475880

[10] Carlson, J., Mazza, N., Thévenaz, J.: Endotrivial modules for $p$-solvable groups. Trans. Amer. Math. Soc. 363, 4979-4996 (2011) Zbl pre05956719 MR 2806698

[11] Carlson, J., Thévenaz, J.: Torsion endotrivial modules. Algebras Represent. Theory 3, 303335 (2000) Zbl 0970.20004 MR 1808129

[12] Carlson, J., Thévenaz, J.: The classification of endo-trivial modules. Invent. Math. 158, 389411 (2004) Zbl 1110.20004 MR 2096798

[13] Carlson, J., Thévenaz, J.: The classification of torsion endotrivial modules. Ann. of Math. (2) 165, 823-883 (2005) Zbl 1110.20005 MR 2183283

[14] Carlson, J., Townsley, L., Valeri-Elizondo, L., Zhang, M.: Cohomology Rings of Finite Groups. With an appendix: Calculations of Cohomology Rings of Groups of Order Dividing 64. Kluwer, Dordrecht (2003) Zbl 1056.20039 MR 2028960

[15] Dade, E. C.: Endo-permutation modules over p-groups, I, II. Ann. of Math. 107, 459-494 (1978), 108, 317-346 (1978) Zbl 0404.16003 MR 0506990

[16] Dade, E. C.: Une extension de la théorie de Hall et Higman. J. Algebra 20, 570-609 (1972) Zbl 0246.20014 MR 0297888

[17] Erdmann, K.: Algebras and quaternion defect groups. I, II. Math. Ann. 281, 545-560, 561582 (1988) Zbl 0655.16011 MR 0958259

[18] Erdmann, K.: Algebras and semidihedral defect groups. I. Proc. London Math. Soc. (3) 57, 109-150 (1988) Zbl 0648.20007 MR 0940432

[19] Erdmann, K., Algebras and semidihedral defect groups. II. Proc. London Math. Soc. (3) 60, 123-165 (1990) Zbl 0687.20006 MR 1023807

[20] Erdmann, K.: Blocks of Tame Representation Type and Related Algebras. Lectures Notes in Math. 1428, Springer (1990) Zbl 0696.20001 MR 1064107

[21] Gorenstein, D.: Finite Groups. Chelsea, New York (1980) Z Zbl 0463.20012 MR 0569209

[22] Mazza, N., Thévenaz, J.: Endotrivial modules in the cyclic case. Arch. Math. (Basel) 89, $497-$ 503 (2007) Zbl 1155.20009 MR 2371685

[23] Olsson, J. B.: On 2-blocks with quaternion and quasidihedral defect groups. J. Algebra 36, 212-241 (1975) Zbl 0314.20009 MR 0376841

[24] Webb, P.: The Auslander-Reiten quiver of a finite group. Math. Z. 179, 97-121 (1982) Zbl 0479.20008 MR 0643050 